\title{
A composite strategy to fabricate high-performance biodegradable stents for tissue regeneration
}

\author{
Jiandong Ding
}

While most of the present medical implants are nonbiodegradable, biodegradable materials are thought to replace many nonbiodegradable ones in the future, which is dependent on development of the corresponding core techniques of biomaterials [1-4]. Much progress has been made in the field of biodegradable materials during the last decade [5-7]. Besides introducing new materials with novel chemical structures, an important strategy might be fabrication of various composites based on the available biomaterials or their derivatives.

A composite could be defined as follows: "Generally speaking, any material consisting of two or more components with different properties and distinct boundaries between the components can be referred to as a composite material" [8]. Krishan K. Chawla [9] gives an operational definition: "We shall call a material that satisfies the following conditions a composite material: 1 . it is manufactured (i.e., naturally occurring composites, such as wood, are excluded); 2. it consists of two or more physically and/or chemically distinct, suitably arranged or distributed phases with an interface separating them; 3 . it has characteristics that are not depicted by any of the components in isolation." So, it could be understood that a composite indicates a material composed of two classes of materials and exhibits new or significantly enhanced properties beneficial for mankind.

Recently, a strategy to fabricate polymer-polymer composite stents was published in Nature Materials coauthored by the group of Maria Palasis from 480 Biomedical, Inc., USA, and also by Robert Langer from Massachusetts Institute of Technology, and George M. Whitesides from Harvard University [10]. They used one polymer to coat another polymer of a different type to enhance the mechanical properties while maintaining biodegradation. These authors employed fibers of biodegradable aliphatic polyesters such as poly(L-lactide-co- glycolide) (L-PLGA) as the base, and introduced an elastomer coating to further make a composite. As shown in Fig. 1a, the elastomer is also based on a biodegradable copolyester such as poly(glycolide-co-caprolactone) (PGCL). A four-arm branched prepolymer was crosslinked by hexamethylene diisocyanate (HDI) to form an elastomer network with urethane crosslinks.

It is surprising that such a facile composite technique enhanced the mechanical property very significantly. While utilizing resorbable polymers, both radial stiffness (RRF) and chronic outward force (COF), as measures of compression and expanding forces, respectively, became similar to those of benchmark self-expanding metallic stents, with some in vitro results shown in Fig. 1b. Such a polymer-polymer composite stents exhibited satisfactory biodegradation and biocompatibility behaviors in vitro and in vivo, with some of results from an ovine model demonstrated in Fig. 1c. The in vivo experiments employed mainly a model of swine ilio-femoral vessels and led to a good vessel repair efficacy.

Endovascular interventional treatment is an approach that deals with the cardiovascular diseases [11]. Stents have been widely used in clinics, especially with combination of the interventional treatment. Biodegradable or bioresorbable stents or scaffolds are thought to be a milestone in coronary intervention [12] and considered to be the third generation, while the first and second generations are bare metal stents and drug-eluting stents. (The new generation could also be drug-eluting, and the main difference between the second and third generations comes from nonbiodegradable versus biodegradable.) Metals are predominant in the first and second generation stents in clinics. It is inspiring to develop a biodegradable scaffold with high mechanical strength and expansion properties similar to classic metallic stents. The commercialized biodegradable polymeric stent is mainly

State Key Laboratory of Molecular Engineering of Polymers, Department of Macromolecular Science, Fudan University, Shanghai 200438, China Email: jdding1@fudan.edu.cn 
a



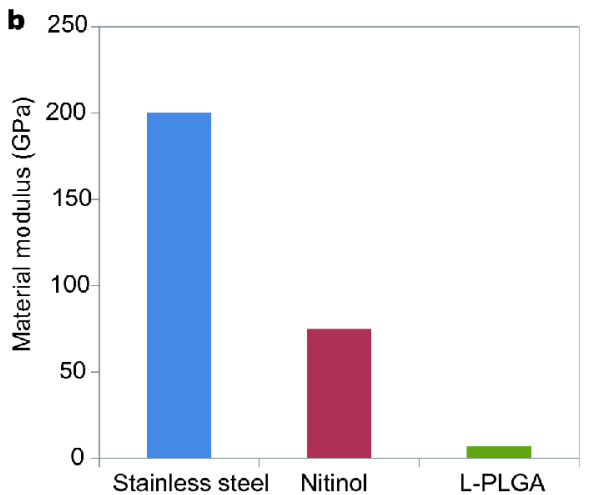

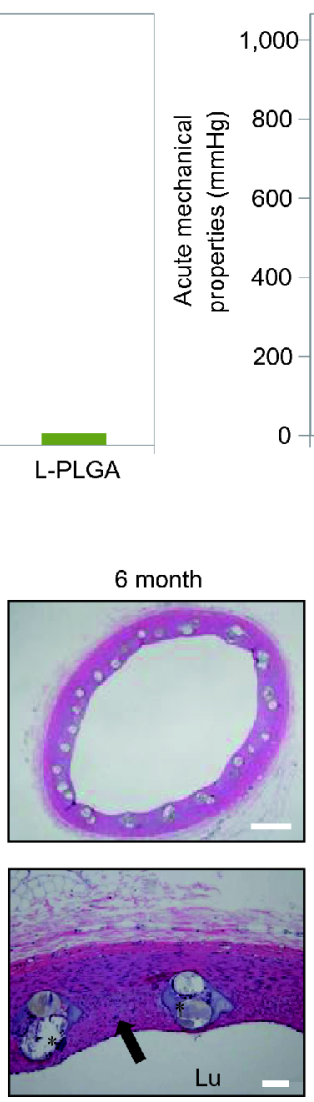
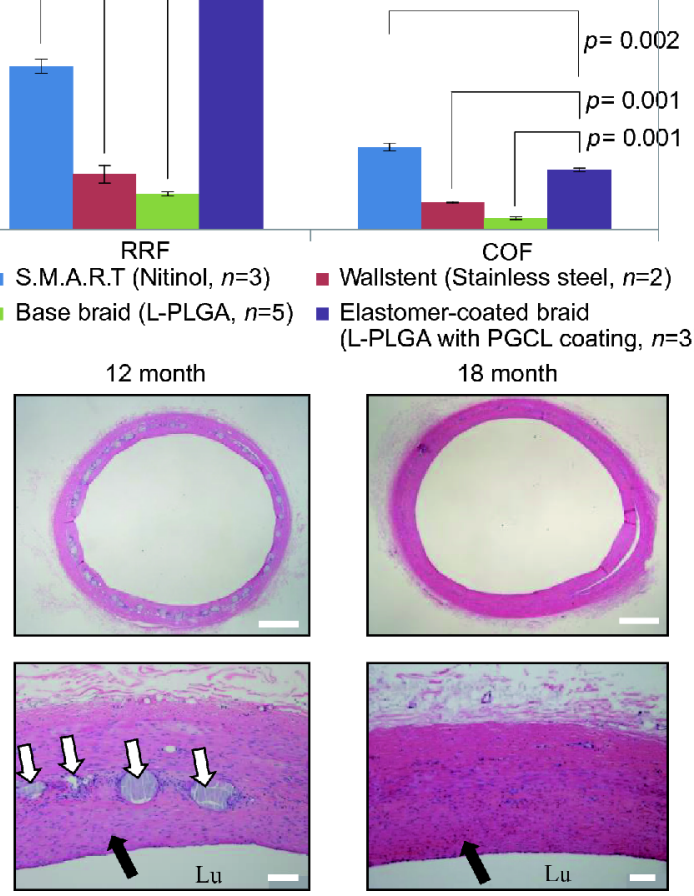

Wallstent (Stainless steel, $n=2$ )

Elastomer-coated braid

(L-PLGA with PGCL coating, $n=3$ )
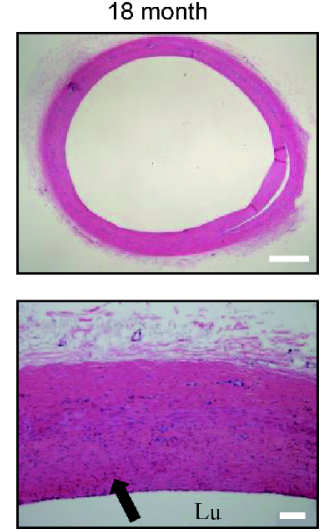

Figure 1 Biodegradable composite polymeric stents with high mechanical strength and biocompatibility. (a) Structure of a stent. (b) Mechanical properties of an elastomer-coated braid (L-PLGA coated with PGCL) in comparison with the Wallstent (stainless steel) and S.M.A.R.T. (Nitinol). (c) Haematoxylin and eosin stained images of PLCL implants in superficial femoral or profunda arteries of sheep; scale bars represent $1 \mathrm{~mm}$ in the upper row and $100 \mu \mathrm{m}$ in the lower row. Adapted from Ref. [10] with permission. Copyright 2018, Nature Publishing Group.

made of polylactide (PLA), but the famous Abbot product Bioabsorbable Vascular Scaffolds (BVS) was retracked from market all over the world in 2017 due to "low commercial sales" after appearance of clinical outcomes at 3 years [13]. And the insufficient mechanical properties of biodegradable polymers compared to the commercialized nonbiodegradable metals are believed to be a key reason [14]. So, the publication in Nature Materials 2018 [10] is very important and in time.

It seems worthy noting that the diameter of the reported fiber or strut in the polymeric composite stent is approximately $125-175 \mu \mathrm{m}$, which is about double of the diameter of Wallstent ( $75 \mu \mathrm{m}$ stainless steel). So, in my opinion, it is still open to further compare the mechanical 
properties under similar strut thickness and mesh size of the polymer-polymer composite stents and other stents and also to check the operational convenience during an interventional treatment in more preclinical experiments of animals and even in clinical trials. Nevertheless, the significant enhancement of the mechanical performance of the braided polymeric stents with an elastic coating is still exciting and encouraging.

Very recently, a Chinese group reports a metal-polymer composite strategy to fabricate biodegradable stents [15]. Under such a circumstance, the mechanical property could be guaranteed owing to the application of iron, but the acceleration of the metal corrosion becomes an alternative bottleneck. The in vitro and in vivo experiments indicated that the iron substrate confirmed good mechanical strength at the early stage and the PLA coating adjusted iron corrosion rates to a clinically meaningful time scale [15]. In such a metal-polymer composite stent (MPS), the PLA coating is not simply a surface layer added to the matrix or substrate, but a degradation-rateadjustment layer to accelerate iron corrosion.

Anyway, composite techniques afford a promising direction to develop the next-generation biodegradable stents. Such a strategy is also stimulating for investigating other material systems or devices where both mechanical properties and degradation profiles should be required.

Received 2 February 2018; accepted 7 February 2018; published online 10 February 2018

1 Wu L, Ding J. In vitro degradation of three-dimensional porous poly(D,L-lactide-co-glycolide) scaffolds for tissue engineering. Biomaterials, 2004, 25: 5821-5830

2 Onuma Y, Serruys PW. Bioresorbable scaffold: the advent of a new era in percutaneous coronary and peripheral revascularization?
Circulation, 2011, 123: 779-797

3 Wiebe J, Nef HM, Hamm CW. Current status of bioresorbable scaffolds in the treatment of coronary artery disease. J Am College Cardiology, 2014, 64: 2541-2551

4 Gao R, Yang Y, Han Y, et al. Bioresorbable vascular scaffolds versus metallic stents in patients with coronary artery disease. J Am College Cardiology, 2015, 66: 2298-2309

5 Pan Z, Ding J. Poly(lactide-co-glycolide) porous scaffolds for tissue engineering and regenerative medicine. Interface Focus, 2012, 2: 366-377

6 Nishio S, Kosuga K, Igaki K, et al. Long-term ( $>10$ years) clinical outcomes of first-in-human biodegradable poly-L-lactic acid coronary stents: igaki-tamai stents. Circulation, 2012, 125: 2343-2353

7 Werner M, Micari A, Cioppa A, et al. Evaluation of the biodegradable peripheral igaki-tamai stent in the treatment of de novo lesions in the superficial femoral artery. JACC-Cardiovascular Interventions, 2014, 7: 305-312

8 Vasiliev VV, Morozov EV. Mechanics and Analysis of Composite Materials. Amsterdam: Elsevier, 2001

9 Chawla KK. Composite Materials. New York: Springer, 2012

10 Sharma U, Concagh D, Core L, et al. The development of bioresorbable composite polymeric implants with high mechanical strength. Nat Mater, 2017, 17: 96-103

11 Lakhan SE, Kaplan A, Laird C, et al. The interventionalism of medicine: interventional radiology, cardiology, and neuroradiology. Int Arch Med, 2009, 2: 27

$12 \mathrm{Wu}$ Y, Shen L, Ge L, et al. Six-month outcomes of the XINSORB bioresorbable sirolimus-eluting scaffold in treating single de novo lesions in human coronary artery. Cathet Cardiovasc Intervent, 2016, 87: 630-637

13 Lafont A, Mennuni MG. What happened to the bioresorbable scaffold concept. Circ Cardiovasc Interv, 2018, 11: e006283

14 Foin N, Nakatani S, Bourantas C, et al. TCT-365 incomplete stent apposition causes high shear flow disturbances and delay in neointimal coverage as a function of strut-to-wall detachment distance. J Am College Cardiology, 2014, 64: B106-B107

15 Qi Y, Qi H, He Y, et al. Strategy of metal-polymer composite stent to accelerate biodegradation of iron-based biomaterials. ACS Appl Mater Interfaces, 2018, 10: 182-192 